4 Korama, $\mathrm{K}$ : 'Secure conference key distribution schemes for conspiracy attacks'. IEICE Tech. Rep., 1992, ISEC91-61, pp. 17-20

5 KOYAMA, K., and OHTA, K.: 'Security of improved identity-based conference key distribution systems'. Proc. of Eurocrypt '88, 1989 pp. $11-19$

\title{
SINGLE AND DOUBLE AMPLIFIED RECIRCULATING DELAY LINES AS FIBRE-OPTIC FILTERS
}

\section{C. Vázquez, B. Vizoso, M. López-Amo and} M. A. Muriel

Indexing terms: Optical filters, Delay lines, Optical fibres

Experimental and theoretical results on single and double amplified recirculating delay lines are presented. Their application as fibre-optic filters are discussed, showing a wide flexibility of design, by appropriate choice of their main parameters: loop length, gain and coupling ratio of the coupler.

Introduction: All-fibre devices have been recently proved to be able to implement signal processing functions such as filtering. pulse-train generation, convolution, correlation, and highspeed matrix multiplication [1].

Our aim is to emphasise the application of all-fibre device as filters. Theoretical analyses of recirculating delay lines including erbium doped fibre amplifiers have been carried ou for this purpose. In this analysis 'incoherent signal processing' is dealt with, i.e. we consider intensities rather than fields, in contrast to a previous coherent analysis [2]. We show different types of filter that can be implemented depending on the design parameters, and we also include additional features of double-ring structures. The two configurations to be considered are shown in Fig. 1, and the obtained experimental results have been successfully compared with theory.
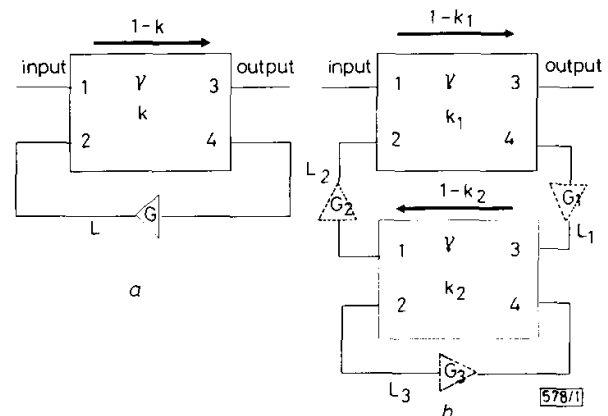

Fig. 1 Configuration of proposed single amplified recirculating delay line and proposed double parallel amplified recirculating delay line

EDFA can be placed within $L_{1}, L_{2}$ or $L_{3}$ (shown in dotted lines) a Single

$b$ Double

Single amplified recirculating delay line: Fig. $1 a$ shows the single recirculating delay line which incorporates a coupler and an erbium doped fibre amplifier with an intensity gain $G$ $I_{1}$ is the input intensity to the coupler and $I_{2}$ is the recirculat ing amplitude in the loop. The power is extracted through port 3 , so the transfer function is defined as $I_{3} / I_{1}$. This discussion deals with linear time-invariant systems (LTISs). The transformation is used, i.e. $z=\exp (j w \tau)$. The transfer function is given as

$$
\begin{array}{r}
H(z)=\left[(1-\gamma)(1-K) z+f G(1-\gamma)^{2}(2 K-1)\right] / \\
{[z-(1-\gamma)(1-K) f G]}
\end{array}
$$

where $\gamma$ and $K$ are the fractional intensity loss and the coupling coefficient of the coupler. $f$ is the transmission coefficient of the fibre, knowing $f=10^{(-a L / 10)}$ where $\alpha$ is the attenuation coefficient in $\mathrm{dB} / \mathrm{m}$ and $L$ the fibre ring length (including the erbium doped fibre). $w \tau=\beta L$ where $\beta$ is the propagation constant and $\tau$ is the delay time through the loop $(\tau=N L / c)$. $N$ is the refractive index of the fibre and $c$ is the velocity of light.

When considering $f=1, \gamma=0$ and defining $K^{*}=1-K$ (to consider port 4 as the output of the configuration, the loop being constructed by connecting ports 3 and 2), we obtain the same expression as in Reference 3 when they assume $L=1$.

Results: A CW LD output of $1.5 \mu \mathrm{m}$ was sinusoidally modulated in frequency by using the tracking generator of an RF spectrum analyser at the desired frequencies. This modulated signal was the input to the recirculating delay line. The setup was constructed with a $2 \times 2, K$ variable polarisationpreserving fibre coupler; its loop length was $40 \mathrm{~m}$, comprising $2 \mathrm{~m}$ of pigtails, and the remainder the EDFA itself, pumped at $1480 \mathrm{~nm}$.

We have centred the measurements on three particular cases, two of them having also been analysed in Reference 3, and an additional one:

(i) Case 1: The zero of the transfer function is placed near -1 , thus

$$
\begin{aligned}
& z_{c}=f G(1-\gamma)\left(1-2 K^{*}\right) / K^{*} \Rightarrow-1 \\
& K^{*}=f G(1-\gamma) /[1+2 f G(1-\gamma)] \\
& p=[f G(1-\gamma)]^{2} /[1+2 f G(1-\gamma)]
\end{aligned}
$$

Fig. $2 a$ shows the ring transfer function for $K^{*}=0 \cdot 2739$, and $G^{*}=f G(1-\gamma) \simeq 0.6058$ (the value of $G^{*}$ comprises not only the gain of the amplifier but also the losses caused by connectors, the transmission of the fibre and the fractional intensity loss of the coupler). The locations of zeros and poles are: $z_{c}=-1, p=0 \cdot 1659$. Theoretically, by selecting an exact $G^{*}$, the differences between high and low amplitudes would be $>70 \mathrm{~dB}$.

(ii) Case 2: The pole is placed close to +1 and the zero near -1 by selecting a certain value of $G$ (in this case the loss dependence disappears completely).

$$
\begin{aligned}
& p=K^{*} G f(1-\gamma) \Rightarrow 1 \\
& G=1 /\left[K^{*} f(1-\gamma)\right] \\
& z_{c} \Rightarrow-1 \\
& G=K^{*} /\left[\left(1-2 K^{*}\right) f(1-\gamma)\right]
\end{aligned}
$$

if eqn. $6=$ eqn. $7, K^{*}=\sqrt{ }(2-1)$ and $G^{*}=\sqrt{ }(2+1)$

Fig. $2 b$ shows the experimental results, with $K^{*}=0.414$, $G^{*}=2.399, p=0.9931, z_{c}=-0.1$. In the laboratory this filtering structure causes problems in attempting to obtain a stable figure close to the unit circle. Such a situation may be caused by light source instability, or environmentally induced loop-length fluctuations, but the main problems are those related to the amplifier itself. The ASE (amplified spontaneous emission) and its associated resonance (in other words, the laser behaviour of the structure [4] when the cavity approaches an overall gain $\left.G_{0}=K^{*}(1-\gamma) f G_{A S E}=1\right)$ have to be avoided. This can be partially avoided by using a filter of several nanometres around the signal wavelength [2]. SBS (stimulated Brioullin backscattering) is overcome by using an optical isolator in the optical amplifier. Finally, it is necessary to stabilise the amplifier gain. This final point causes fluctuations in $G$ that obviate the possibility of working on the unit circle without going out of it, leading to an unstable transfer function (oscillator). It is also important to note that in the dynamic range we are working within, the input signals have to be of high enough magnitude to avoid ASE but not so high that the amplifier reaches its saturation region.

(ii) Case 3: Fig. $2 c$ shows the optimised case, with the pole close to +1 and the zero far from -1 . The data for Fig. $2 c$ 
are: $K^{*}=0.6, G^{*}=1.664 ; p=0.9984, z_{c}=-0.5546$. When trying to reach the unit circle, the same problems as described in case 2 were encountered.

Double parallel amplified recirculating delay line: The configuration is shown in Fig 16 . The analysis is based on the same terms as for the single recirculating delay line, so we only discuss the stability conditions of the structure.

Three conditions are required when considering stability, but they are not sufficient to enable a complete investigation. It would be also necessary to analyse the temporal response of the system or to study how small fluctuations in the different
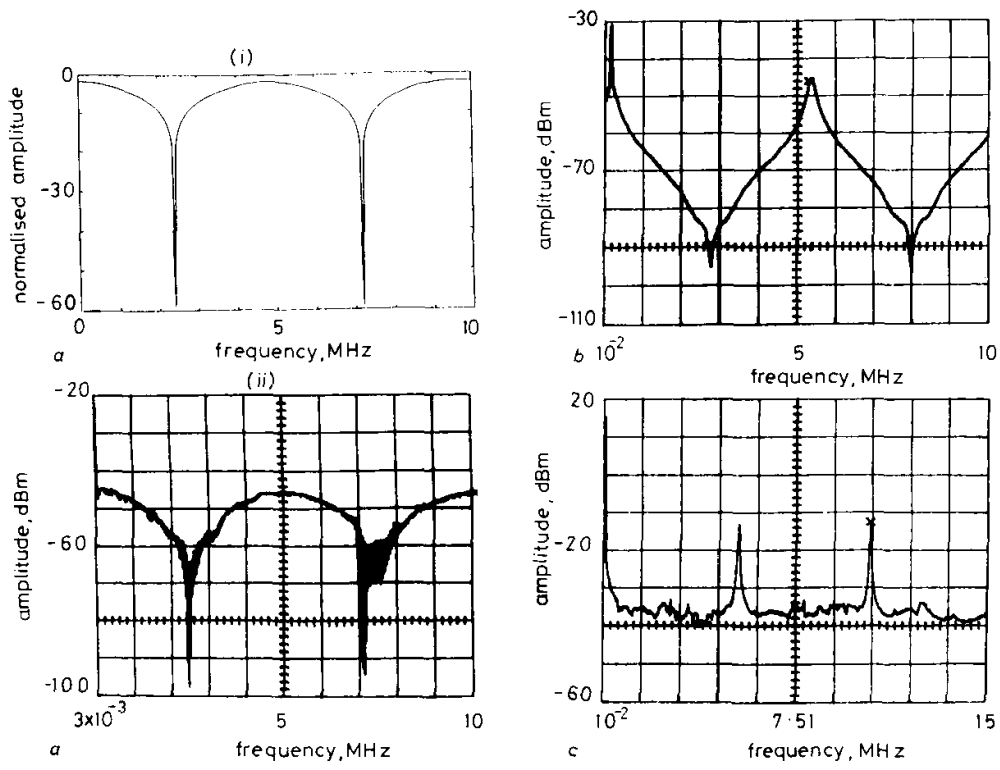

Fig. 2 Single amplified recirculating delay line

$a$ Case $1: z_{c}=-1, p=0 \cdot 1659, K^{*}=0 \cdot 2739, G^{*}=0 \cdot 6058$

(i) calculations

(ii) experimental results

$b$ Case 2: experimental results for $z_{c}=-1, p=0.9931, K^{*}=0.414, G^{*}=2.399$

$c$ Case 3: experimental results for $z_{c}=-0.5546, p=0.9984, K^{*}=0.6, G^{*}=1.664$
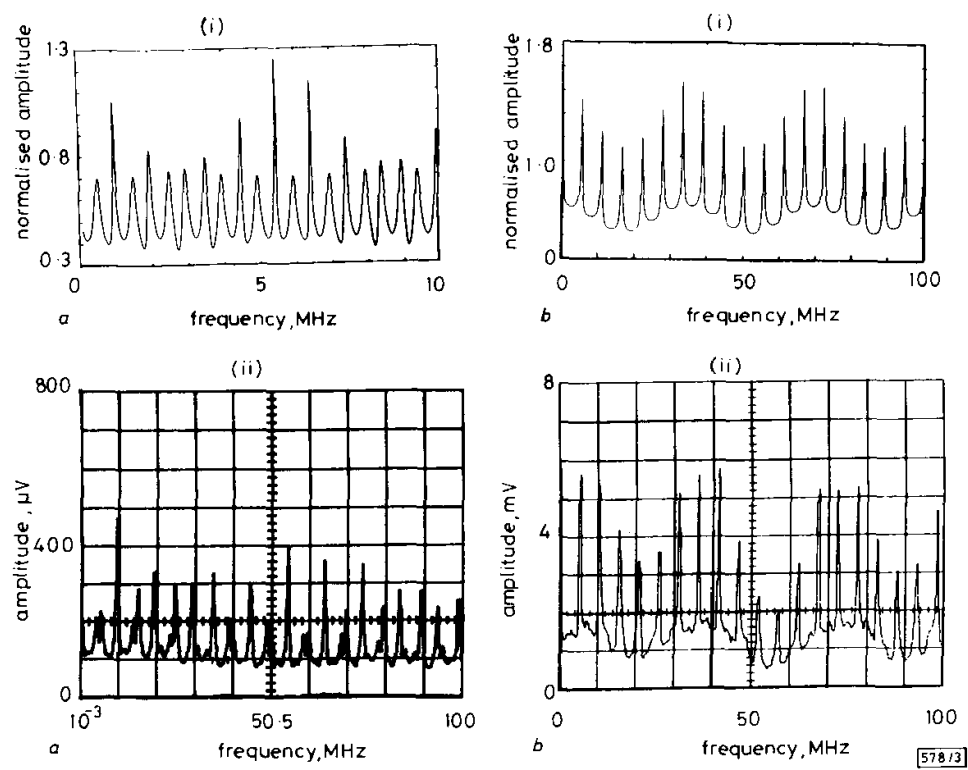

Fig. 3 Transfer functions of double parallel amplified recirculating delay line

a EDFA in $\mathrm{L}_{2}, K_{1}=0.457, K_{2}=0.4227, L_{1}=2 \mathrm{~m}, L_{3}=22 \mathrm{~m}, G=1.5, \alpha=0.3 \times 10^{-3} \mathrm{~dB} / \mathrm{m}, \gamma=0.05$

(i) calculations

(ii) experimental results

b EDFA in $L_{3} ; K_{1}=0.9, K_{2}=0.4, L_{1}=4 \mathrm{~m}, L_{2}=2 \mathrm{~m}, G_{3}=1.44, \alpha=0.3 \times 10^{-3} \mathrm{~dB} / \mathrm{m}, \gamma=0.05$

(i) calculations

(ii) experimental results 
parameters can alter the stability of the system. Those three conditions are:

$$
\begin{aligned}
G_{1}^{*} G_{2}^{*} & \leq 1 /\left[\left(1-K_{1}\right)\left(1-K_{2}\right)\right] \\
G_{3}^{*} & \leq 1 /\left(1-K_{2}\right) \\
G_{1}^{*} G_{2}^{*} G_{3}^{*} & \leq 1 /\left[K_{2}^{2}\left(1-K_{1}\right)\right]
\end{aligned}
$$

where $G_{i}^{*}=f_{i} G_{i}(1-\gamma), i=1,2,3$.

Some measurements have been taken, and they have been successfully tested with theory (Fig. 3), showing their capability of reshaping the spectral response because of the additional parameters they offer compared to single structures.

Conclusions: Double and single amplified recirculating delay lines have been described theoretically and experimental results have been reported. These configurations have successfully shown their flexibility of design to obtain different profiles depending on the choice of certain parameters: gain, loop length, and coupling ratios of the couplers. High peak to peak amplitudes and FWHM can be achieved. Different cases, to reflect the diversity of its possibilities, have been analysed. Their performance would be further improved by eliminating environmental hazards and stabilising the amplifier gain. Further analysis of double and more complex amplified recirculating structures can lead to a wider range of applications.

Acknowledgment: We wish to thank R. Civera for his help in software development and J. Capmany for his helpful discussions. We also thank ATT. This work was supported by the Spanish CICYT (TIC-211/89), CAM-PRI (CO59/90) and UPM.

3Ist March 1992

M. C. Vázquez B. Vizoso, M. López-Amo and M. A Murie Mepartamento de Tecnología Fotónica. ETSI de Telecomunicación, (Departamento de Tecnologia Fotonica. ET
Ciudad Universitaria, 28040-Madrid, Spain)

\section{References}

I JACKsOn, K. P, et al.: 'Fiber optic delay line signal processing', IEEE Trans., 1985, MTT-33, (3), pp. 193-210

2 OKAMURA, H., and IWATSUKI, K.: 'A finesse-enhanced Er-doped fiber ring resonator', J. Lightwave Technol., 1991, 9, (11), pp. 15541560

3 MOSLEHI, B.: 'Fibre-optic filters employing optical amplifier to provide design flexibility', Electron. Lett., 1992, 3, pp. 226-228

4 URQUHART, P.: 'Review of rare earth doped fibre lasers and amplifiers', IEE Proc. J, 1989, 135, (6), pp. 385-407

\section{DUAL-DEVICE ACTIVE PATCH ANTENNA WITH IMPROVED RADIATION CHARACTERISTICS}

\section{R. A. York and R. C. Compton}

Indexing terms: Antennas, Microstrip, Gunn oscillators

An active microstrip patch antenna for quasioptical powercombining arrays is described. The oscillator circuit uses two Gunn diodes which symmetrically load a resonant patch. The symmetry of the structure and the push-pull operation The symmet polarisation, improved copolarisation patterns, and twice as polarisation, improved copolarisation
much power as a single-device patch.

Introduction: Arrays of coupled microwave and millimetrewave oscillators offer an attractive method for combining the power from many solid-state devices. Such arrays have been demonstrated with a variety of different devices [1], and have been recently mode-locked for high-power pulse generation [2]. Each element of the array is a selfcontained oscillator, built around a semiconductor device and a planar antenna.
Several different microwave oscillator circuits based on a microstrip patch antenna have appeared in the literature using Gunn devices [3-4], IMPATTs [5-6], and FETs [7-8]. Millimetre-wave circuits using Gunn devices and slo antennas have also been reported [9]. The patch antenna is useful because it is simple to fabricate, can easily accommodate devices, and serves as a resonant stabilisation element in the oscillator circuit. In practice, however, active patch antennas with devices integrated directly inside the patch suffer very high crosspolarisation fields [3]. We report a modified active patch antenna, which is symmetrically loaded with two Gunn devices. This effects a significant reduction in crosspolarisation losses, results in more symmetrical copolarisation radiation patterns, and has the added advantage of doubling the available power from each array element.

Description: A commonly used active patch antenna circuit for two-terminal devices is shown in Fig. 1a. A Gunn diode

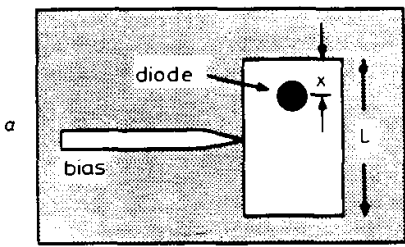

grounded dlelectric subs trate



62411

Fig. 1 Microstrip patch antenna loaded with single, active two-terminal device [3-4] and symmetrical, two-diode patch, which improves crosspolarisation and doubles the power

a Microstrip patch antenna

$b$ Two-diode

(or IMPATT) is integrated within the patch, shunting the top metallisation and the ground plane. The patch is designed to operate in the lowest-order resonant mode, with DC bias supplied to the device at null in the electric field under the patch. The design of these circuits proceeds semi-empirically, with the device located where the input resistance of the patch equals the negative resistance of the device. For a halfwavelength patch, the diode location can be found from the simple expression [4]

$$
\frac{x}{L}=\frac{1}{\pi} \cos ^{-1}\left[\frac{0 \cdot 123 W}{\lambda_{0}} \sqrt{ }\left(R_{d}\right)\right]
$$

where $W$ is the patch width and $R_{d}$ is the magnitude of the device negative resistance. The reactive impedance of the device will effect a change in the resonance frequency of the patch.

When the diode package occupies an appreciable fraction of the path volume (the usual case at $X$-band or higher) the actual current distribution on the patch will be quite different to that of a similar but deviceless patch. An excess of higher order current modes will be generated on the patch to account for this discontinuity. These higher-order modes are partially responsible for the high crosspolarisation observed in such active patch antennas. Asymmetry in the device location (again, the usual case) will further exacerbate this problem by exciting current modes with odd symmetry, resulting in asymmetrical copolar patterns. Another major contribution to the $\mathrm{H}$-plane crosspolarisation arises from the vertical currents in the diode between the patch and ground plane (the 'feed' radiation).

Some of these problems can be alleviated through the use of a second diode, which is located diametrically opposite the

ELECTRONICS LETTERS 21st MaY 1992 Vol. 28 No. 11 